\title{
Simple Controlling Ecofriendly Synthesis of Silver Nanoparticles at Room Temperature Using Lemon Juice Extract and Commercial Rice Vinegar
}

\author{
T. Lan Anh Luu (D, X. Truong Cao, V. Thai Nguyen, N. Linh Pham, H. Lam Nguyen, \\ and C. Tu Nguyen iD \\ School of Engineering Physics, Hanoi University of Science and Technology, No. 1, Dai Co Viet Street, 100000 Hanoi, Vietnam \\ Correspondence should be addressed to T. Lan Anh Luu; anh.luuthilan@hust.edu.vn and C. Tu Nguyen; tu.nguyencong@ \\ hust.edu.vn
}

Received 2 October 2019; Revised 11 March 2020; Accepted 10 April 2020; Published 19 May 2020

Academic Editor: Paresh Chandra Ray

Copyright (c) 2020 T. Lan Anh Luu et al. This is an open access article distributed under the Creative Commons Attribution License, which permits unrestricted use, distribution, and reproduction in any medium, provided the original work is properly cited.

\begin{abstract}
Silver nanoparticles were prepared in an ecofriendly manner at room temperature via the stepwise-modified Tollens route using the lemon juice extract and commercial rice vinegar. In this work, the lemon juice extract-a natural-origin chemical—was used as a reducing and stabilizing agent, and commercial rice vinegar was used to create a low acidic environment to control the silver nanoparticle growth via the stepwise method. The average dimension of silver nanoparticles was qualitatively evaluated through the UV-Vis spectra via the Mie theory. The X-ray diffraction and field emission scanning electron spectroscopy were employed to study the purity, the crystal structure, and the morphology of samples, respectively. Due to the weak activity and low purity of ecofriendly chemicals, the reaction and baking times strongly affect the preparation efficiency in obtaining small-size silver nanoparticles $(\sim 40 \mathrm{~nm})$. The highest efficiency was obtained with $24 \mathrm{~h}$ reaction time and $48 \mathrm{~h}$ baking time. The bimodal distribution of the size of silver nanoparticles was observed by UV-Vis analysis and field emission scanning electron microscopy. The obtained small-size silver nanoparticles $(\sim 40 \mathrm{~nm})$ have a uniform dimension. The quality of the obtained silver nanoparticles was evaluated through the conducting properties of silver paint made from ecosynthesized silver nanoparticles which showed a promising prospect to develop green-synthesized silver paint working at room temperature.
\end{abstract}

\section{Introduction}

The silver nanostructures, especially silver nanoparticles (Ag NPs), have attracted much attention for several decades due to their outstanding properties such as high antibacterial activity, antifungal activity, high conductivity, and plasmonic property [1-4]. These outstanding properties of Ag NPs have been applied in many applications such as in textile, food packaging, health care, electronics, and chemical industries [4-11]. Recently, the interest in Ag nanostructures is once more bloomed due to the applicability of Ag NPs in brand-new applications such as food additives [12], nanomedicine and biomedical applications [13-16], and surface-enhanced Raman scattering substrates for a biosensor [17-21]. To prepare Ag NPs, many routes have been used such as chemical, physical, and biomedical methods $[1,4,16,22-26]$ which have similar steps in preparing and preserving Ag NPs in a solution. In these methods, the chemical route is the most widely used method in which ion $\mathrm{Ag}^{+}$originated from silver salt is reduced by different reducing agents such as acetate (Turkevich method) or sodium borohydride $\mathrm{NaBH}_{4}$ (Brust-Schiffrin synthesis), plant extract, or natural-origin chemical $[3,27]$.

Recently, due to the demand of using Ag NPs in bio and medical applications and the urge for sustainable development, the green and ecofriendly synthesis process in which nontoxic and environmentally safe chemicals are 
used as reducing agents attracts huge attention $[13,25-31]$. For example, Abidin et al. used the apple extract to synthesize Ag NPs and studied their antibacterial properties [28]; Bagherzade et al. using the saffron extract to prepare Ag NPs for antibacterial applications [29]; Tippayawat et al. studied the antibacterial activity of Ag NPs synthesized using the aloevera plant extract [30]; Singh et al. prepared Ag NPs using sun-dried tulsi leaves and studied the applicability of obtained Ag NPs in reducing 4-nitrophenol [32]; Prathna et al. studied Ag NPs synthesized using the Citrus limon (lemon) aqueous extract [33]. Besides the bio and medical applications, the green and ecofriendly synthesis are also developed to prepare Ag NPs for higher-quality applications such as silver ink, smart electronics, and catalyst [34-37]. Chen et al. used glucose to green synthesize Ag NPs at $80^{\circ} \mathrm{C}$ to enhance the conductivity of conductive ink [35]. Sampaio et al. used the artichoke aqueous extract to green synthesize Ag NPs at $40^{\circ} \mathrm{C}$ and studied their electrical conductivity [36]. Li et al. prepared $\mathrm{Ag} \mathrm{NPs}$ at room temperature and studied their applicability in conductive ink [37]. These works, except for the work of $\mathrm{Li}$ et al., required complicated conditions during the preparation process-stabilizing the temperature of precursor solutions. Moreover, the drawing patterns using green-synthesized Ag NPs have to be processed by UV sintering [35], heating at $100^{\circ} \mathrm{C}[36]$, or infrared sintering [37] to get stable results. These sintering treatments will limit the applicability of green-synthesized Ag NP-based conductive ink. To overcome this limit, recently, the conductive ink which could work at room temperature is preferably developed.

Our previous work shows that citric acid has an important role in both controlling the size of NPs and enhancing the efficiency of Ag NP fabrication [38]. To make the synthesis ecofriendlier, we aim to develop a preparation process in which industrial products are replaced by natural-origin products or domestic products. In this paper, we report a simple process to control the ecofriendly synthesis of Ag NPs at room temperature based on the stepwise-modified Tollens method. In this process, the lemon juice extract-a natural-origin citric acid-is used as a reducing agent and stabilizing agent, and commercial rice vinegar (acetic acid) is used to regulate the acidity for controlling the Ag NP growth based on the LaMer mechanism. The size distribution and the preparation efficiency are theoretically estimated via the Mie theory [39] using UV-Vis data. Because of the weak activity and low purity of the lemon juice extract and commercial rice vinegar, the reaction time and the baking time strongly affect the Ag NPs' preparation efficiency. The reaction time and baking time are varied to find the optimal synthesis condition. The purity of obtained Ag NP powder is examined through $\mathrm{X}$-ray diffraction analysis. The quality of obtained Ag NPs was also qualitatively evaluated through testing the conductivity at room temperature of silver paint made from the obtained Ag NP powder. The formation mechanism of Ag NPs is also discussed in detail.

\section{Materials and Methods}

2.1. Sample Preparation. Lemons purchased at a local market were extracted by a lemon squeezing tool. The juice was filtered by $15-\mu \mathrm{m}$ pore filter paper and then stored in the refrigerator. The rice vinegar is commercial (Vinegar TOAN THU, CNC Hanoi company). Other chemicals were at an analytical grade and used without any further purification. A buffer acid solution was prepared by mixing commercial rice vinegar, formic acid, and bidistilled water with the volume ratio of $38: 12: 50$ under magnetic stirring. The silver nanoparticle preparation process was carried out as follows (Figure 1): $10 \mathrm{ml}$ of $\mathrm{AgNO}_{3} 0.15 \mathrm{M}$ was mixed with $50 \mathrm{ml}$ of lemon juice extract and $4.5 \mathrm{ml}$ ammonia solution (28\%) to get a precursor solution of $\mathrm{pH}=8.59$; it was stirred for $15 \mathrm{~min} ; 10 \mathrm{ml}$ of prepared buffer acid solution was added into the precursor solution to carry out stepwise process; the solution was stirred vigorously during different reaction times $(6,24$, and $48 \mathrm{~h})$; after completing the reaction step, the obtained suspension was put into an ultrasonic bath to perform ultrasonic treatment for 10 minutes, and this treatment was repeated two more times; after three times of ultrasonic treatment, Ag NPs were centrifuged at an angular speed of $4500 \mathrm{rev} / \mathrm{min}$ (rpm) for 20 minutes to obtain the asprepared slurry; the as-prepared slurry was put into an oven at $80^{\circ} \mathrm{C}$ for baking treatment at different times $(24,48$, and $72 \mathrm{~h}$ ); after baking treatment, the obtained product-Ag NP powder-was dispersed in ethanol and cleaned using centrifugal treatment at an angular speed of $4500 \mathrm{rpm}$ for 20 mins; the cleaned slurry was then dispersed into bidistilled water to obtain final Ag NP suspension for further study. In this work, to study the effect of reaction time on asprepared Ag NPs (before baking treatment), a small part of the as-prepared slurry was dispersed in bidistilled water to get as-prepared Ag NP suspension.

To prepare the silver paint, a solvent was firstly prepared by mixing ethanol (Et), triton X-100 (TX), ethylene glycol (EG), and isopropyl alcohol (IPA) with a weight percent of 4.7, 9.4, 62.7, and 3.2\%, respectively. The as-prepared Ag NP powder was dispersed in the prepared solvent with a Ag weight percent of $20 \%$. The obtained suspension was processed with ultrasonic treatment for 20 minutes to get silver paint. The silver paint was then put into a commercial $0.7 \mathrm{~mm}$ rollerball pen lead for conductivity testing.

2.2. Analytical Methods. The UV-Vis spectroscopy of samples was observed with a Varian $100 \mathrm{UV}-\mathrm{V}$ is spectrometer. The X-ray diffraction (XRD) pattern of Ag NP powder was recorded using an X'pert Pro (PANalytical) powder X-ray diffractometer operated at a voltage of $40 \mathrm{kV}$ and current of $15 \mathrm{~mA}$ using $\mathrm{CuK} \alpha$ radiation $\left(\lambda=1.54065 \mathrm{~A}^{\circ}\right)$ with the angle range from 20 to $75^{\circ}$ and a scanning rate of $0.03^{\circ}$ s. Morphological analysis of the Ag NPs was performed using field-emission scanning electron microscopy (FESEM, JEOL JSM-7600F). To prepare the sample for FESEM measurement, the final Ag NP suspension was dropped on the $\mathrm{SiO}_{2} / \mathrm{Si}$ substrate and then dried in the oven at $80^{\circ} \mathrm{C}$ for $24 \mathrm{~h}$. 


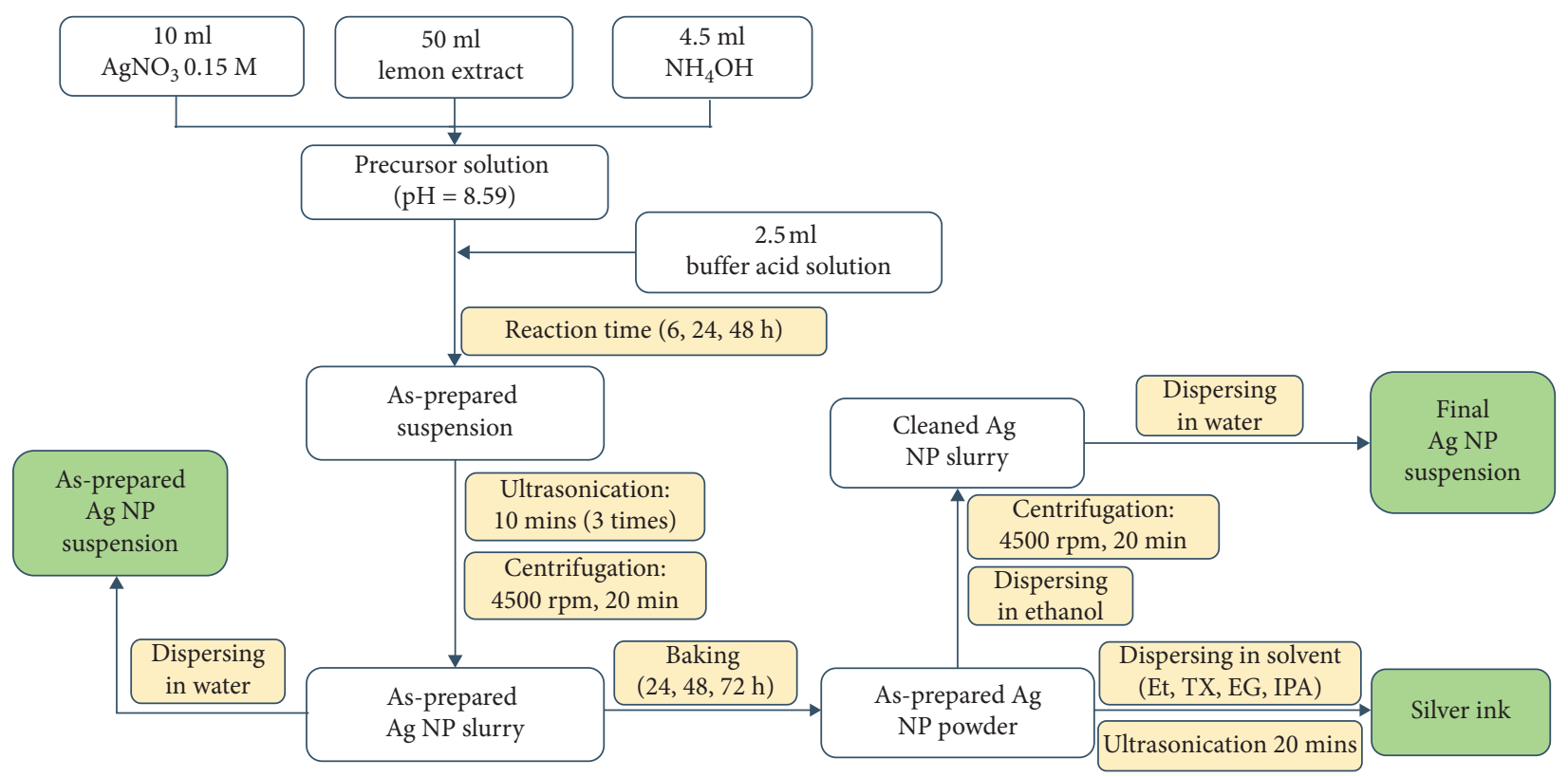

FIGURE 1: Schematic diagram of the preparation process of silver nanoparticles using the lemon extract and rice vinegar.

\section{Results and Discussion}

3.1. Effect of Reaction Time. Due to the use of natural-origin and ecofriendly chemicals that have inevitably unwanted chemicals, the reduction process will take a longer time to complete than compared with using analytical-grade chemicals in the traditional route. To find a proper reaction condition, the Ag NP preparation efficiency was examined with different reaction times. After finishing the reaction step, the as-prepared slurry separated from the as-prepared solution was dispersed into bidistilled water to get as-prepared Ag NP suspension. Figure 2(a) demonstrates the UVVis spectra of as-prepared Ag NP suspensions with different reaction times $(6,24$, and $48 \mathrm{~h})$. All UV-Vis spectra of asprepared Ag NP suspensions have a similar pattern as of Ag NP suspension in published works [39-41] which qualitatively confirmed the appearance of Ag NPs in the as-prepared suspension. In the UV-Vis spectra, there is the main peak at around $430 \mathrm{~nm}$ and a wide shoulder at around $570 \mathrm{~nm}$, and there is no absorption peak for the lemon fruit extract (Figure S1(a)) and rice vinegar (Figure S1(b)), respectively. To study further the origin of the peak and wide shoulder, Lorentz deconvolution was used to analyze the UV-Vis spectra. Figure 2(b) manifests the Lorentz deconvolution of the UV-Vis spectrum of as-prepared Ag NP suspension synthesized with $24 \mathrm{~h}$ reaction time. The results of the Lorentz deconvolution of all as-prepared Ag NP suspensions are listed in Table 1 , in which $R^{2}$ is Adj. $R$-square of Lorentz deconvolution and $A_{1} / A_{2}$ is the ratio between the area of peak 1 and peak 2 .

The results in Table 1 imply that with $6 \mathrm{~h}$ reaction time, there is only one large peak with low intensity in the spectra. The wide full width at half maximum (FWHM) and low UVVis signal could be the results of the incompletion of the reduction process. With $24 \mathrm{~h}$ and $48 \mathrm{~h}$ reaction times, UVVis spectra are deconvoluted into two peaks: peak1 at around $426 \mathrm{~nm}$ and peak 2 at around $560 \mathrm{~nm}$. Peak 1 and peak 2 are assigned to the signal of small $(<100 \mathrm{~nm})$ [3, 39, 42] and big (hundreds nm) [3, 42, 43] Ag NPs, respectively. When reaction time increases from $24 \mathrm{~h}$ to $48 \mathrm{~h}$, the position of peak 1 does not change (remains at $426.4 \mathrm{~nm}$ ) but the position of peak 2 shifts from $569.5 \mathrm{~nm}$ to $563.9 \mathrm{~nm}$, and the ratio between the area of peak 1 and peak 2 decreases from 1.1 to 0.5 . The unchanging of peak 1 's position at $426.4 \mathrm{~nm}$ implies that the reaction completes after $24 \mathrm{~h}$, and prepared Ag NPs have an average dimension of approximately $55 \mathrm{~nm}$ via the Mie theory [39]; from $24 \mathrm{~h}$ to $48 \mathrm{~h}$, the small Ag NPs coalesce to form larger Ag NPs following the Ostwald ripening mechanism [44] which reduces the ratio between the area of peak 1 and peak 2 . From these results, we choose the reaction time which gives the highest efficiency of small Ag NP preparation which is $24 \mathrm{~h}$ for further studies.

3.2. Effect of Baking Time. To completely remove the byproduct from Ag NPs and to obtain Ag NP powder for further study such as XRD and FESEM analysis, the asprepared Ag NP slurries were baked at $80^{\circ} \mathrm{C}$. Due to the complicated effect of the baking treatment [45], another task in our research is to study the effect of baking time on the characteristic of Ag NP powders. Figure 3(a) presents the XRD patterns of Ag NP powders obtained after different baking times-24, 48 , and $72 \mathrm{~h}$. The XRD patterns of all three samples have the same pattern which hints that baking time does not affect the crystal structure of Ag NPs.

For further study of the crystal properties and purity of samples, the HighScore Plus software was used to analyze the XRD patterns. The results of XRD analysis show that there is more than one compound of silver in the as-prepared Ag NP powder. In the XRD pattern of as-prepared Ag NP powder, the appearance of the XRD patterns of silver (ICSD card No. 98-018-0878) and silver oxide AgO (ICSD card No. 00-003- 


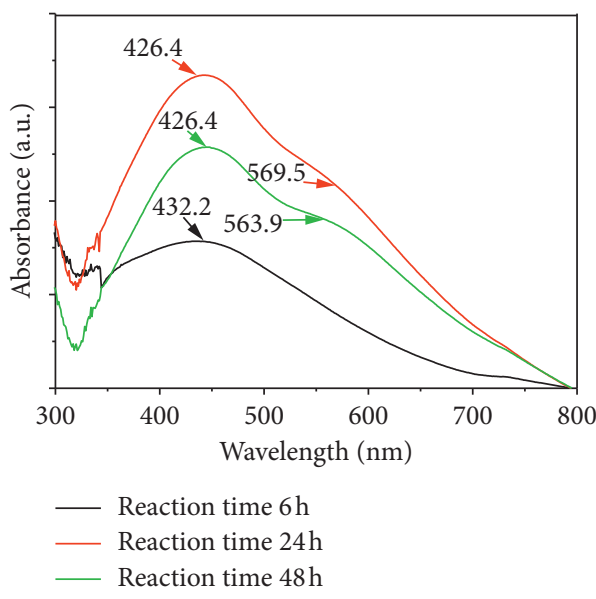

(a)

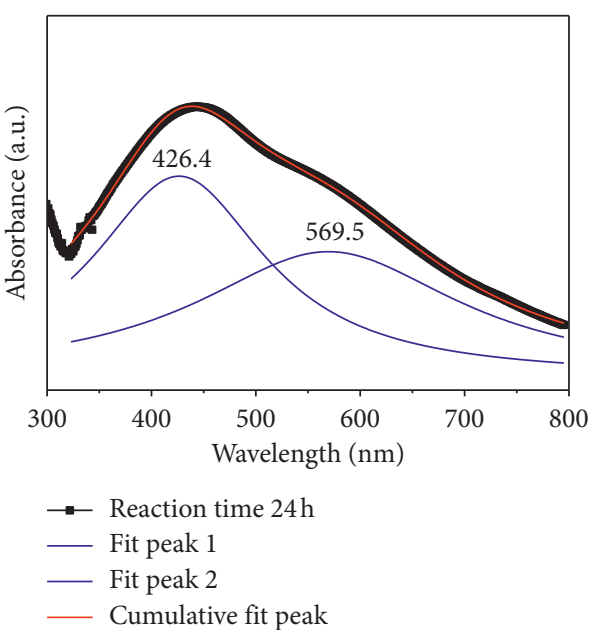

(b)

FIgURE 2: The UV-Vis spectra of samples at different reaction times.

TABLE 1: The position, width, and area of peak 1 and peak 2 determined from Lorentz deconvolution of UV-Vis spectra of samples prepared with different reaction times.

\begin{tabular}{|c|c|c|c|c|c|c|c|c|}
\hline \multirow[b]{2}{*}{ Reaction time } & \multicolumn{3}{|c|}{ Peak 1} & \multicolumn{3}{|c|}{ Peak 2} & \multirow[b]{2}{*}{$R^{2}$} & \multirow[b]{2}{*}{$A_{1} / A_{2}$} \\
\hline & Wavelength (nm) & FWHM (nm) & $\begin{array}{l}\text { Area of peak } 1 \\
\left(A_{1}\right) \text { (a.u.) }\end{array}$ & Wavelength (nm) & FWHM & $\begin{array}{c}\text { Area of peak } 2 \\
\left(A_{2}\right) \text { (a.u.) }\end{array}$ & & \\
\hline $6 \mathrm{~h}$ & 432.2 & 323.0 & 216.9 & & & & 0.99 & \\
\hline $24 \mathrm{~h}$ & 426.4 & 202.2 & 221.5 & 569.5 & 308.1 & 210.7 & 0.99 & 1.1 \\
\hline $48 \mathrm{~h}$ & 426.4 & 164.6 & 124.3 & 563.9 & 362.7 & 266.6 & 0.99 & 0.5 \\
\hline
\end{tabular}

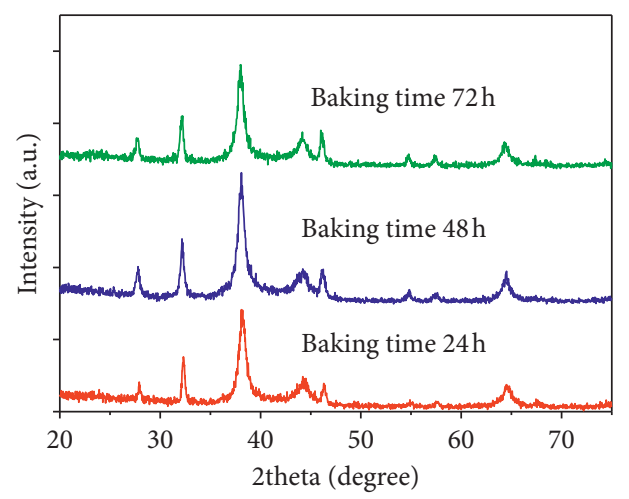

(a)

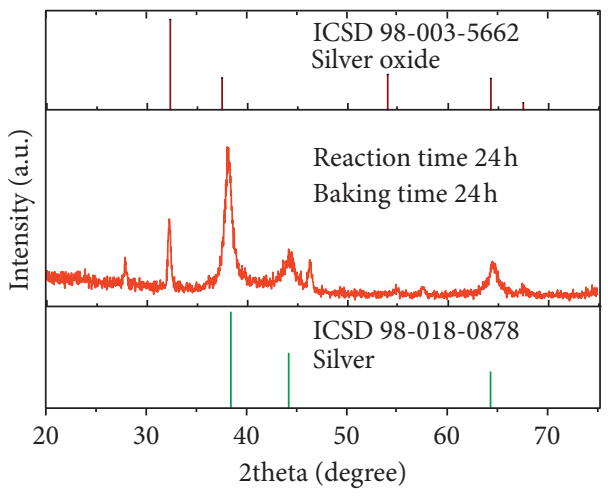

(b)

Figure 3: Continued. 


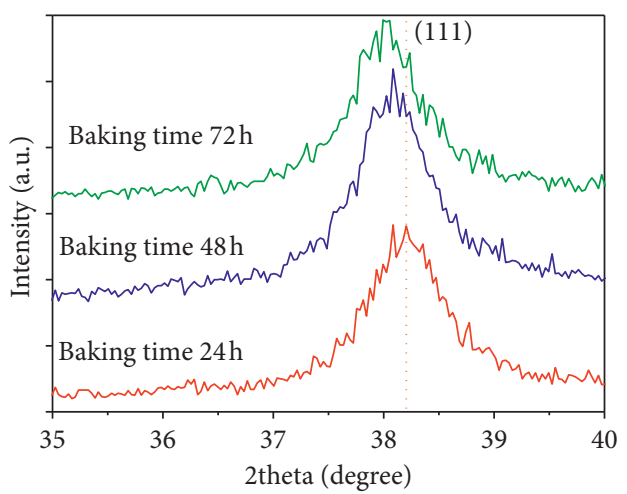

(c)

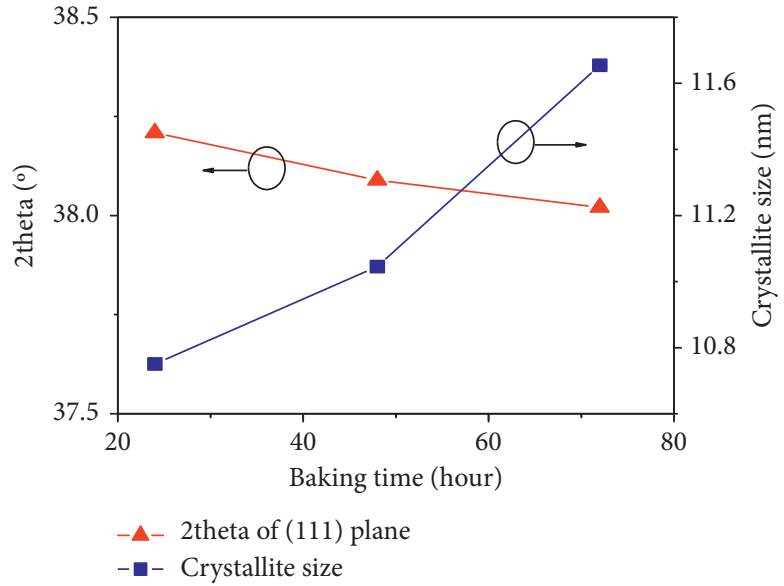

(d)

FIGURE 3: (a) The XRD pattern of Ag NP powders; (b) XRD pattern of the sample baking for $24 \mathrm{~h}$ in comparison with the standard pattern of the silver (ICSD 98-018-0878) and silver oxide AgO (ICSD 00-003-5662); (c) the XRD patterns of samples focusing on the peak (111) of Ag; (d) the variation of position of the diffraction peak of the (111) plane and the crystallite size of Ag with baking time.

5662 ) is observed in which most signal originates from silver (Figure 3(b)). These results confirm the successful preparation of Ag NPs using the lemon juice extract and rice vinegar. The appearance of silver oxide in the product might originate from the oxidation during the baking in ambient air. Besides the XRD patterns of silver and silver oxide, there are two peaks at $27.85^{\circ}$ and $46.45^{\circ}$ which are assigned to the organic compounds of silver with chemical in the lemon juice extract and commercial rice vinegar (the appearance of these organic compounds is also confirmed with energy dispersive spectroscopy-Figure S2). The XRD analysis also shows that the diffraction peak of the (111) plane of silver (ICSD card No. 98-018-0878) is shifted to a lower diffraction angle when baking time increases (Figure 3(c)). This shift hints the increase of the crystallite size of Ag with baking time which is also confirmed by crystallite size calculation using the Scherrer formula as follows:

$$
d=\frac{0.9 \times \lambda}{\beta \times \cos \theta}
$$

where $d$ is the average crystallite size, $\theta$ is the diffraction angle, $\beta$ is the full-width at half maximum of the strongest peak-peak of the (111) plane, and $\lambda=0.154065 \mathrm{~nm}$ is the wavelength of X-ray of $\mathrm{CuK} \alpha$. The average crystallite size of as-prepared powder increases from 10.7 to 11.0 and $11.6 \mathrm{~nm}$ when the baking time increases from $24 \mathrm{~h}$ to 48 and $72 \mathrm{~h}$, respectively. The increase in the average crystallite size might be assigned as the reason for the increase in the silver grain size observed in UV-Vis analysis.

To evaluate the grain size of Ag NPs, Ag NP powder is cleaned and then dispersed into distilled water to get the final Ag NP suspension (Figure 1). Figure 4 presents the UV-Vis spectra of the final Ag NP suspensions prepared with different baking times 24,48 , and $72 \mathrm{~h}$. The results of the Lorentz deconvolution of UV-Vis spectra are listed in Table 2. The results show that when baking time is longer, both peak 1 and 2 shift to longer wavelength which implies the larger particles

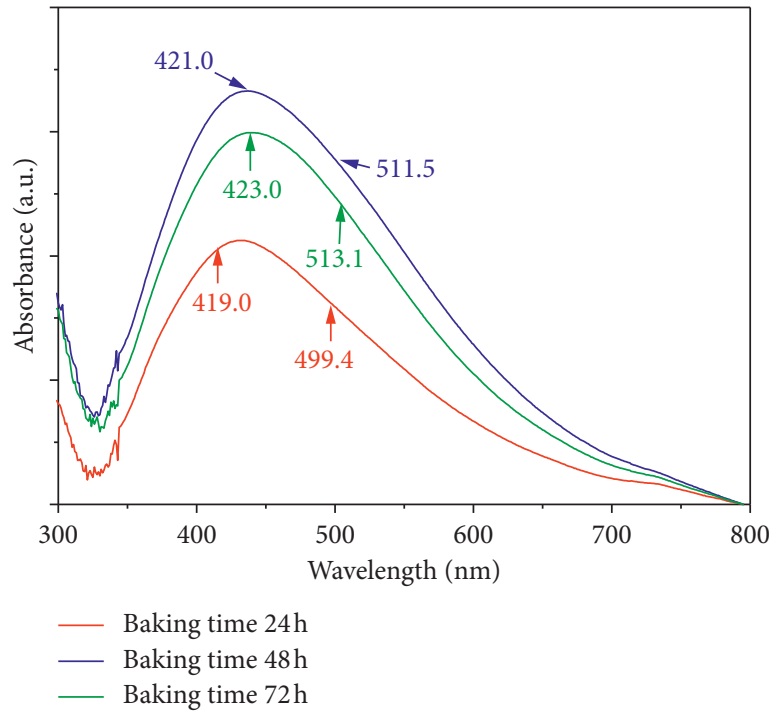

FIgUre 4: The UV-Vis spectra of samples after different baking times.

in suspension via the Mie theory $[39,46]$. When baking time increases from $24 \mathrm{~h}$ to $48 \mathrm{~h}$ and $72 \mathrm{~h}$, peak 1 shifts from $419.0 \mathrm{~nm}$ to 421.0 and $423.0 \mathrm{~nm}$ and peak 2 shifts from $499.4 \mathrm{~nm}$ to 511.5 and $513.1 \mathrm{~nm}$, respectively. With all studied baking times, the position of peak 1 is in the region which is equivalent to the size of Ag NPs in the range of approximately $50 \mathrm{~nm}$ [39]. Moreover, after baking, the position of peak 1 and peak 2 is shifted to lower wavelength (426.4 and $569.5 \mathrm{~nm}$ in Section 3.1) and the full-width at half maximum of UV-Vis spectra in the range from 320 to $700 \mathrm{~nm}$ is shrunk. The reason might be the removal of by-products during the baking process. The $A_{1} / A_{2}$ ratio increases from 0.53 to 0.59 and then decreases to 0.54 when the baking time increases from $24 \mathrm{~h}$ to $48 \mathrm{~h}$ and then $72 \mathrm{~h}$, respectively. From these results, we choose the $48 \mathrm{~h}$ baking time sample for further research. 
TABLE 2: The position, width, and area of peak 1 and peak 2 determined from Lorentz approximation of UV-Vis spectra of samples in baking times.

\begin{tabular}{|c|c|c|c|c|c|c|c|c|}
\hline \multirow{2}{*}{ Baking time } & \multicolumn{3}{|c|}{ Peak 1} & \multicolumn{3}{|c|}{ Peak 2} & \multirow{2}{*}{$R^{2}$} & \multirow{2}{*}{$A_{1} / A_{2}$} \\
\hline & Wavelength (nm) & Area of peak 1 (a.u.) & FWHM & Wavelength (nm) & Area of peak 2 (a.u.) & FWHM & & \\
\hline $24 \mathrm{~h}$ & 419.0 & 53.2 & 100.4 & 499.4 & 100.6 & 208.1 & 0.98 & 0.53 \\
\hline $48 \mathrm{~h}$ & 421.0 & 110.1 & 134.5 & 511.5 & 185.3 & 234.5 & 0.99 & 0.59 \\
\hline $72 \mathrm{~h}$ & 423.0 & 100.0 & 130.9 & 513.1 & 185.0 & 261.2 & 0.99 & 0.54 \\
\hline
\end{tabular}

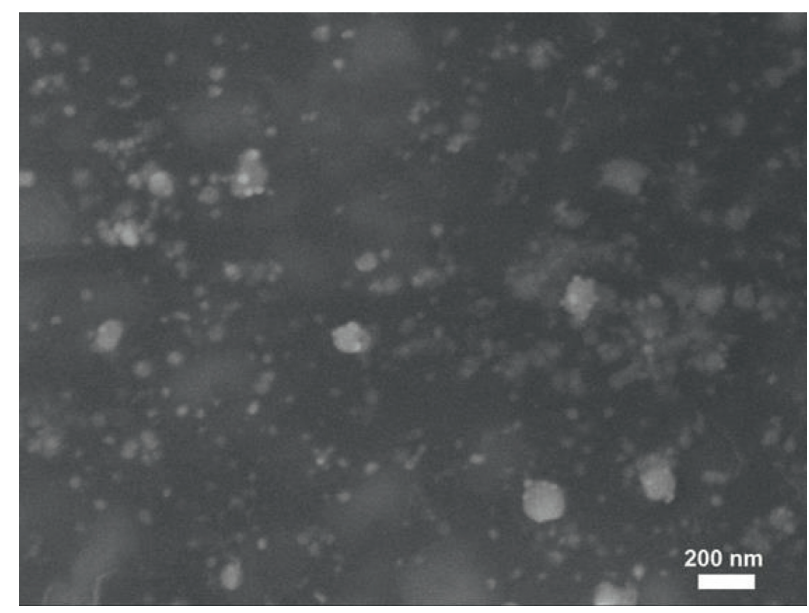

Figure 5: The FESEM image of the Ag NP powder sample coated on the $\mathrm{SiO}_{2} / \mathrm{Si}$ substrate.

3.3. Morphology of AgNPs. For visual observation of Ag NPs, the Ag NP suspension was dropped on the $\mathrm{SiO}_{2} / \mathrm{Si}$ substrate and then dried at $80^{\circ} \mathrm{C}$ in ambient air for $24 \mathrm{~h}$. Figure 5 presents the FESEM image of samples prepared with $24 \mathrm{~h}$ reaction time and $48 \mathrm{~h}$ baking time. FESEM analysis shows that after drying, Ag NPs have two main morphologies of Ag NPs: small $(\sim 40 \mathrm{~nm})$ and large $(\sim 150 \mathrm{~nm})$ NPs. This result is in good accordance with the two-peak pattern observed in the UV-Vis analysis in the previous section. The difference between the theoretical value observed from the UV-Vis data and the value observed from the FESEM analysis might be due to the effect of the surfactant in the solution. The opaque parts in the FESEM image are assigned to the remaining surfactant in the Ag NP suspension. The FESEM result also implies that the small Ag NPs have uniform size even after baking. This uniformity is an expecting property for many applications of Ag NPs.

3.4. The Formation Mechanism of Ag NPs. The formation mechanism of Ag NPs is explained mainly via the LaMer mechanism or the stepwise method [47], in which the preparation process is divided into two main stages: nucleation and growth. The nucleation and growth stages occur mainly at high and low $\mathrm{pH}$, respectively. In the nucleation stage, the reductant plays a vital role, but in the growth stage, the stabilizers play a key role; however, in this work, citric acid in the lemon juice extract plays both key roles. Based on the LaMer mechanism, in the synthesis approach of the metal nanoparticle using plant extracts, there are three main phases: activation phase, growth phase, and termination phase [48-51]. The first phase is the activation phase in which the reduction of metal ions takes place followed by nucleation steps of the already reduced metal atoms. The second phase is the growth phase, where the small-sized NPs spontaneously combine with others to form a larger-sized particle. This process is as Ostwald ripening, in which thermodynamic stability of nanoparticles also gets increased. The third phase is the termination phase, which determines the nanoparticle's final shape [48-51]. The formation mechanism of nanoparticles may be explained in detail as follows (Figure 6): first, silver ions react with citric acid-lemon juice extract-in high $\mathrm{pH}$ environment $(\mathrm{pH}=8.59)$ to create the seeds of Ag NPs, when the concentration of silver seeds in the solution reaches supersaturation, and nucleation burst takes place; in the subsequent step, the appearance of rice vinegar in the buffer acid solution promotes the growth stage in which small-size Ag NPs coalesce together to form larger-size Ag NPs; in the final step, the lemon juice extract having bioactive polyphenols, alkaloids, flavonoids, ascorbic acid, etc., acts as the stabilizers to form Ag nanoparticles.

3.5. The Applicability of Ag NPs to Silver Ink. In our work, to verify the quality of ecosynthesized Ag NPs for high-quality applications such as silver ink and smart electronics, the obtained Ag NP powder was used to prepare silver ink. The Ag slurry obtained after cleaning with ethanol was dispersed into the solvent of Et, TX, EG, and IPA (Figure 7(a)) to prepare silver ink. The ink was written down and traced three times (Figure 7(b)). The ink dries quickly after writing. 


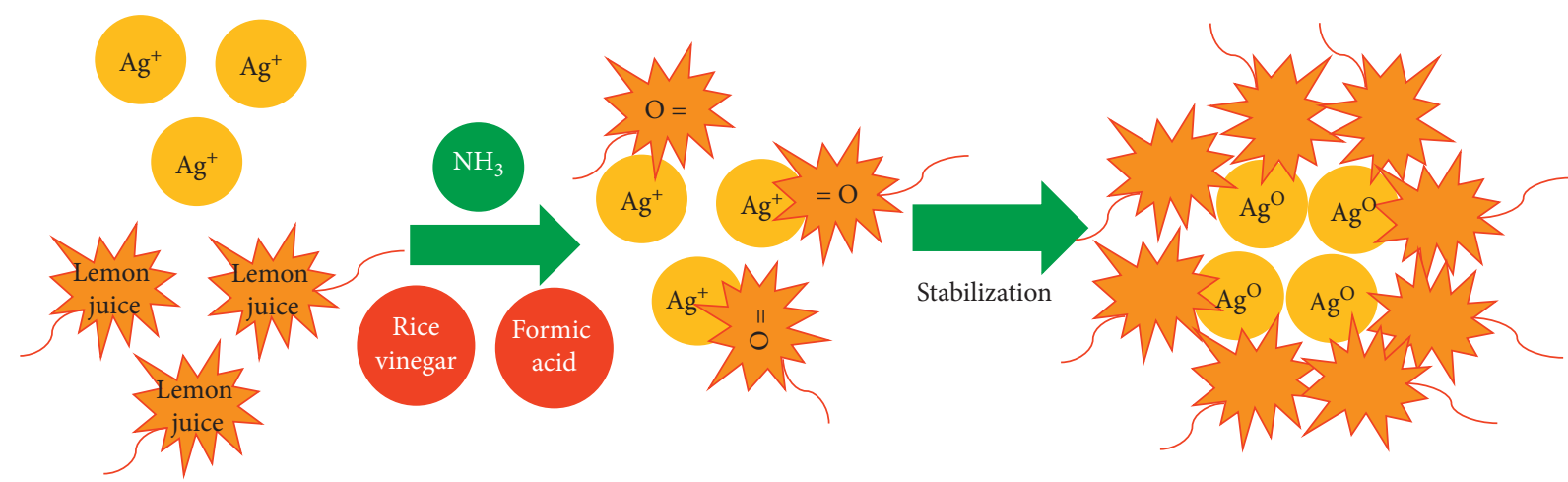

FIGURE 6: The mechanism diagram of the preparation process of silver nanoparticles using the lemon juice extract and rice vinegar.

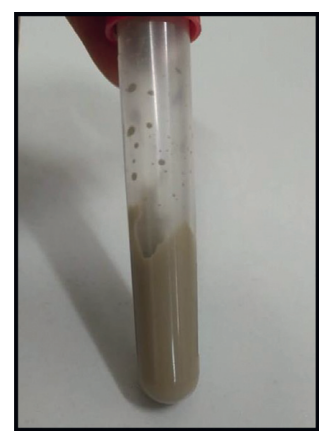

(a)

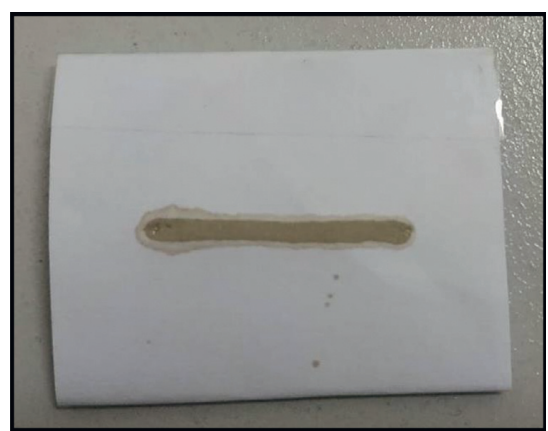

(b)

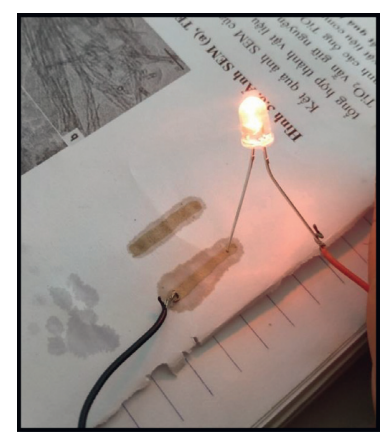

(c)

Figure 7: (a) Photo of as-prepared silver paint, (b) a line of silver paint drawn on an A4 paper (70 gram) using a $0.7 \mathrm{~mm}$ rollerball pen, and (c) conductivity test using a line of silver paint as a conductor in a circuit to light an LED.

To test the characteristic of silver ink, the line was used as a connected wire in a circuit (Figure $7(\mathrm{c})$ ). As written down, a bias voltage is applied for the circuit and the LED light in 10 seconds. These results are promising to develop silver conductive ink working at room temperature in ambient air by the ecofriendly route.

\section{Conclusions}

Ag NPs with two-modal size distribution (40 nm and $200 \mathrm{~nm}$ ) were synthesized in an ecofriendly manner via the stepwise-modified Tollens route at room temperature using the lemon juice extract and commercial rice vinegar. The appearance of Ag NPs was confirmed by UV-Vis and XRD analysis. The mean grain size of Ag NPs was theoretically estimated using the Mie theory which is in good accordance with the FESEM result. Due to the weak activity and low purity of the lemon juice extract and commercial rice vinegar, the effect of reaction time and baking time on the Ag NP preparation process was studied. With $24 \mathrm{~h}$ reaction time, the preparation process shows the highest efficiency in obtaining small-size Ag NP preparation (smaller than $50 \mathrm{~nm}$ ). Baking treatment at $80^{\circ} \mathrm{C}$ in ambient air was used to remove the by-product which also caused the coalescence of small Ag NPs via the Oswald ripening mechanism. With $48 \mathrm{~h}$ baking time, the preparation process gets the highest efficiency in obtaining small Ag NPs. The formation mechanism of Ag NPs was explained via the LaMer mechanism in which the lemon juice extract plays as both reducing and stabilizing agents. The silver ink made from Ag NPs obtained with $24 \mathrm{~h}$ reaction time and $48 \mathrm{~h}$ baking time showed high potential to develop green-synthesized silver ink working at room temperature.

\section{Data Availability}

The processed data (in the origin files) used to support the findings of this study are available from the corresponding authors upon request.

\section{Conflicts of Interest}

The authors declare that they have no conflicts of interest.

\section{Supplementary Materials}

Figure S1: absorbance spectra of (a) lemon extract and (b) commercial rice vinegar. Figure S2: the energy dispersive spectroscopy of as-prepared Ag NP powder (reaction time of $24 \mathrm{~h}$, baking time of $48 \mathrm{~h}$ ). The large content of carbon is due to the carbon tape used in measurement. (Supplementary Materials) 


\section{References}

[1] V. K. Sharma, R. A. Yngard, and Y. Lin, "Silver nanoparticles: green synthesis and their antimicrobial activities," Advances in Colloid and Interface Science, vol. 145, no. 1-2, pp. 83-96, 2009.

[2] N. Chauke and F. K. Siebrits, "Evaluation of silver nanoparticles as a possible coccidiostat in broiler production," South African Journal of Animal Science, vol. 42, no. 5, pp. 493-497, 2012.

[3] S. Lee and B.-H. Jun, "Silver nanoparticles: synthesis and application for nanomedicine," International Journal of Molecular Sciences, vol. 20, no. 4, p. 865, 2019.

[4] S. P. Deshmukh, S. M. Patil, S. B. Mullani, and S. D. Delekar, "Silver nanoparticles as an effective disinfectant: a review," Materials Science and Engineering: C, vol. 97, pp. 954-965, 2019.

[5] O. V. Mikhailov, "Synthesis of Ag nanoparticles under a contact of water solution with silver(I)chloride biopolymer matrix," Journal of Molecular Liquids, vol. 291, Article ID 111354, 2019.

[6] Shahid-ul-Islam, B. S. Butola, A. Gupta, and A. Roy, "Multifunctional finishing of cellulosic fabric via facile, rapid insitu green synthesis of AgNPs using pomegranate peel extract biomolecules," Sustainable Chemistry and Pharmacy, vol. 12, Article ID 100135, 2019.

[7] R. Chokkareddy, N. Thondavada, B. Kabane, and G. G. Redhi, "Current advances in biosynthesis of silver nanoparticles and their applications," Macabresque Human Violation and Hate Genocide, Mass Atrocity Enemy-Making, pp. 167-198, Scrivener Publishing LLC, Beverly, MA, USA, 2018.

[8] J. Bárta, L. Procházková, V. Vaněček, M. Kuzár, M. Nikl, and V. Čuba, "Photochemical synthesis of nano- and microcrystalline particles in aqueous solutions," Applied Surface Science, vol. 479, pp. 506-511, 2019.

[9] R. Kotcherlakota, S. Das, and C. R. Patra, Therapeutic Applications of Green-Synthesized Silver Nanoparticles, Elsevier Inc., Amsterdam, Netherlands, 2019.

[10] N. Krishna, G. N. Kumar, T. Neethu, R. John, S. R. Babu, and S. Smitha Chandran, "One pot green synthesis of silver nanoparticles with multiple applications," Materials Today: Proceedings, vol. 5, no. 9, pp. 20567-20571, 2018.

[11] C.-W. Chang, T.-Y. Cheng, and Y.-C. Liao, "Encapsulated silver nanoparticles in water/oil emulsion for conductive inks," Journal of the Taiwan Institute of Chemical Engineers, vol. 92, pp. 8-14, 2018.

[12] I. Abad-Âlvaro, C. Trujillo, E. Bolea et al., "Silver nanoparticles-clays nanocomposites as feed additives: characterization of silver species released during in vitro digestions. Effects on silver retention in pigs," Microchemical Journal, vol. 149, Article ID 104040, 2019.

[13] K. S. Siddiqi, A. Husen, and R. A. K. Rao, "A review on biosynthesis of silver nanoparticles and their biocidal properties," Journal of Nanobiotechnology, vol. 16, no. 14, pp. 1-28, 2018.

[14] A.-C. Burdusel, O. Gherasim, A. M. Grumezescu, L. Mogoanta, A. Ficai, and E. Andronescu, "Biomedical applications of silver nanoparticles: an up-to-date overview," Nanomaterials, vol. 8, p. 681, 2018.

[15] Q. H. Tran, V. Q. Nguyen, and A.-T. Le, "Silver nanoparticles: synthesis, properties, toxicology, applications and perspectives," Advances in Natural Sciences: Nanoscience and Nanotechnology, vol. 4, no. 3, Article ID 033001, 2013.
[16] X. F. Zhang, Z. G. Liu, W. Shen, and S. Gurunathan, "Silver nanoparticles: synthesis, characterization, properties, applications, and therapeutic approaches," International Journal of Molecular Sciences, vol. 17, no. 9, 2016.

[17] Y. Guo, J. Yu, C. Li et al., "SERS substrate based on the flexible hybrid of polydimethylsiloxane and silver colloid decorated with silver nanoparticles," Optics Express, vol. 26, no. 17, pp. 21784-21796, 2018.

[18] A. P. Craig, A. S. Franca, and J. Irudayaraj, "Surface-enhanced Raman spectroscopy applied to food safety," Annual Review of Food Science and Technology, vol. 4, no. 1, pp. 369-380, 2013.

[19] C. Wei, M. Li, and X. Zhao, "Surface-enhanced Raman scattering (SERS) with silver nano substrates synthesized by microwave for rapid detection of foodborne pathogens," Frontiers in Microbiology, vol. 9, pp. 1-9, 2018.

[20] X. Shen, K. Han, L. Ma, M. Gao, X. Xu, and J. Luo, "Nano-Agforest based surface enhanced Raman spectroscopy (SERS) of confined acetic acid," Colloids and Surfaces A: Physicochemical and Engineering Aspects, vol. 547, pp. 126-133, 2018.

[21] J. Zheng and L. He, "Surface-enhanced Raman spectroscopy for the chemical analysis of food," Comprehensive Reviews in Food Science and Food Safety, vol. 13, no. 3, pp. 317-328, 2014.

[22] A. Haider and I. K. Kang, "Preparation of silver nanoparticles and their industrial and biomedical applications: a comprehensive review," Advances in Materials Science and Engineering, vol. 2015, pp. 1-16, 2015.

[23] J. Natsuki, "A review of silver nanoparticles: synthesis methods, properties and applications," International Journal of Materials Science and Applications, vol. 4, no. 5, p. 325, 2015.

[24] D. Tien, L. Chen, N. Van Thai, and S. Ashraf, "Study of Ag and $\mathrm{Au}$ nanoparticles synthesized by arc discharge in deionized water," Journal of Nanomaterials, vol. 2010, Article id 634757, pp. 1-9, 2010.

[25] M. Rawat, "A review on green synthesis and characterization of silver nanoparticles and their applications: a green nanoworld," World Journal of Pharmacy and Pharmaceutical Sciences, vol. 5, no. 7, pp. 730-762, 2016.

[26] J. Singh, T. Dutta, K. H. Kim, M. Rawat, P. Samddar, and P. Kumar, "Green' synthesis of metals and their oxide nanoparticles: applications for environmental remediation," Journal of Nanobiotechnology, vol. 16, no. 1, pp. 1-24, 2018.

[27] M. G. Guzman, J. Dille, and S. Godet, "Antibacterial activity of silver nanoparticles prepared by a chemical reduction method," International Journal of Chemical and Biomolecular Engineering, vol. 2, no. 3, pp. 104-111, 2009.

[28] R. Yahya, Z. A. Ali, S. D. Sekaran, and R. Puteh, "Green synthesis of silver nanoparticles using apple extract and its antibacterial properties," Advances in Materials Science and Engineering, vol. 2016, Article id 4102196, pp. 1-6, 2016.

[29] G. Bagherzade, M. M. Tavakoli, and M. H. Namaei, "Green synthesis of silver nanoparticles using aqueous extract of saffron (Crocus sativus L.) wastages and its antibacterial activity against six bacteria," Asian Pacific Journal of Tropical Biomedicine, vol. 7, no. 3, pp. 227-233, 2017.

[30] P. Tippayawat, N. Phromviyo, P. Boueroy, and A. Chompoosor, "Green synthesis of silver nanoparticles in aloe vera plant extract prepared by a hydrothermal method and their synergistic antibacterial activity," PeerJ, vol. 4, Article ID e2589, 2016.

[31] S. K. Srikar, D. D. Giri, D. B. Pal, P. K. Mishra, and S. N. Upadhyay, "Green synthesis of silver nanoparticles: a review," Green and Sustainable Chemistry, vol. 6, no. 1, pp. 34-56, 2016. 
[32] J. Singh, A. Mehta, M. Rawat, and S. Basu, "Green synthesis of silver nanoparticles using sun dried tulsi leaves and its catalytic application for 4-Nitrophenol reduction," Journal of Environmental Chemical Engineering, vol. 6, no. 1, pp. 1468-1474, 2018.

[33] T. C. Prathna, N. Chandrasekaran, A. M. Raichur, and A. Mukherjee, "Biomimetic synthesis of silver nanoparticles by Citrus limon (lemon) aqueous extract and theoretical prediction of particle size," Colloids and Surfaces B: Biointerfaces, vol. 82, no. 1, pp. 152-159, 2011.

[34] L. Nayak, S. Mohanty, S. K. Nayak, and A. Ramadoss, "A review on inkjet printing of nanoparticle inks for flexible electronics," Journal of Materials Chemistry C, vol. 7, no. 29, pp. 8771-8795, 2019.

[35] Q. Chen, G. Liu, G. Chen, T. Mi, and J. Tai, "Green synthesis of silver nanoparticles with glucose for conductivity enhancement of conductive ink," BioResources, vol. 12, no. 1, pp. 608-621, 2017.

[36] S. Sampaio and J. C. Viana, "Production of silver nanoparticles by green synthesis using artichoke (Cynara scolymus L.) aqueous extract and measurement of their electrical conductivity," Advances in Natural Sciences: Nanoscience and Nanotechnology, vol. 9, no. 4, pp. 045002-045010, 2018.

[37] W. Li, Q. Cen, W. Li et al., "A green method for synthesizing novel nanoparticles and their application in flexible conductive patterns," Journal of Materiomics, vol. 6, no. 2, pp. 300-307, 2020.

[38] M. S. Ho, T. L. A. Luu, H. L. Nguyen, and C. T. Nguyen, "Size control of Ag nanoparticles by green synthesis," in Proceedings of the 9th International Workshop on Advanced Materials Science and Nanotechnology (IWAMSN 2018), Ninh Binh, Vietnam, pp. 364-369, November 2018.

[39] D. Paramelle, A. Sadovoy, S. Gorelik, P. Free, J. Hobley, and D. G. Fernig, "A rapid method to estimate the concentration of citrate capped silver nanoparticles from UV-visible light spectra," The Analyst, vol. 139, no. 19, pp. 4855-4861, 2014.

[40] Y. Cai, X. Piao, W. Gao, Z. Zhang, E. Nie, and Z. Sun, "Largescale and facile synthesis of silver nanoparticles via a microwave method for a conductive pen," RSC Advances, vol. 7, no. 54, pp. 34041-34048, 2017.

[41] O. Pawar, N. Deshpande, S. Dagade, S. Waghmode, and P. Nigam Joshi, "Green synthesis of silver nanoparticles from purple acid phosphatase apoenzyme isolated from a new sourceLimonia acidissima," Journal of Experimental Nanoscience, vol. 11, no. 1, pp. 28-37, 2016.

[42] X. Dong, X. Ji, H. Wu, L. Zhao, J. Li, and W. Yang, "Shape control of silver nanoparticles by stepwise citrate reduction," The Journal of Physical Chemistry C, vol. 113, no. 16, pp. 6573-6576, 2009.

[43] O. A. D. Gallardo, R. Moiraghi, M. A. Macchione et al., "Silver oxide particles/silver nanoparticles interconversion: susceptibility of forward/backward reactions to the chemical environment at room temperature," RSC Advances, vol. 2, no. 7 , p. 2923, 2012.

[44] P. W. Voorhees, "The theory of Ostwald ripening," Journal of Statistical Physics, vol. 38, no. 1-2, pp. 231-252, 1985.

[45] S. D. Solomon, M. Bahadory, A. V. Jeyarajasingam, S. A. Rutkowsky, C. Boritz, and L. Mulfinger, "Synthesis and study of silver nanoparticles," Journal of Chemical Education, vol. 84, no. 2, pp. 322-325, 2007.

[46] S. Baset, H. Akbari, H. Zeynali, and M. Shafie, "Size measurement of metal and semiconductor nanoparticles via UVVis absorption spectra," Digest Journal of Nanomaterials and Biostructures, vol. 6, no. 2, pp. 709-716, 2011.
[47] V. K. Lamer and R. H. Dinegar, "Theory, production and mechanism of formation of monodispersed hydrosols," Journal of the American Chemical Society, vol. 72, no. 11, pp. 4847-4854, 1950.

[48] S. Patra and R. Madhuri, "Green synthesis of noble metal nanoparticles: a step forward to economical and sustainable development," Macabresque Human Violation and Hate Genocide, Mass Atrocity Enemy-Making, pp. 555-602, Scrivener Publishing LLC, Beverly, MA, USA, 2018.

[49] R. Chokkareddy and G. G. Redhi, "Green synthesis of metal nanoparticles and its reaction mechanisms," Green Metal Nanoparticles, pp. 113-139, Scrivener Publishing LLC, Beverly, MA, USA, 2018.

[50] H. I. Abdel-Shafy and M. S. M. Mansour, "Green synthesis of metallic nanoparticles from natural resources and food waste and their environmental application," Green Metal Nanoparticles, pp. 321-385, Scrivener Publishing LLC, Beverly, MA, USA, 2018.

[51] V. Dhapte and V. Pokharkar, Nanosystems for Drug Delivery: Design, Engineering, and Applications, Elsevier Inc., Amsterdam, Netherlands, 2019. 